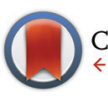

CrossMark

Cite this: Food Funct., 2016, 7, 1884 \section{syndrome; a randomised, controlled trial in an at-risk population \\ Orange juice consumption and its effect on blood lipid profile and indices of the metabolic}

\author{
E. J. Simpson, ${ }^{\star a}$ B. Mendis ${ }^{b}$ and I. A. Macdonald ${ }^{a}$
}

Data from epidemiological and in vitro studies suggest that orange juice (OJ) may have a positive impact on lipid metabolism. However, there have been reports in the media claiming detrimental consequences of $100 \%$ juice consumption, including weight-gain and adverse effects on insulin sensitivity and blood lipid profile. The effect of daily OJ consumption was assessed using a randomised, placebo-controlled, single-blinded, parallel group design. Thirty-six overweight, but otherwise healthy men (40-60 years; 27-35 $\mathrm{kg} \mathrm{m}^{-2}$ ) with elevated fasting serum cholesterol $\left(5-7 \mathrm{mmol} \mathrm{l}^{-1}\right)$, were recruited from the general UK population. None were using nutritional strategies or medication to lower their cholesterol, nor were regular consumers of citrus products. Assessment of BMI, HOMA-IR, and circulating lipid (total cholesterol, low-density lipoprotein, high-density lipoprotein, non-esterified fatty acids, triacylglycerol, apolipoprotein-A1 and apolipoprotein-B) concentrations, was made when fasted before (V1) and after a 12-week intervention (V2), during which participants consumed $250 \mathrm{ml}$ per $\mathrm{d}$ of $\mathrm{OJ}$ or an energy and sugarsmatched orange-flavoured drink (control). The two groups were matched at V1 with respect to all parameters described above. Although triacylglycerol concentration was similar between the groups at both visits, a trend for the change in this variable to differ between groups was observed $(P=0.060)$, with those in control exhibiting a significant increase in triacylglycerol at V2, compared with V1. In OJ, those with the highest initial triacylglycerol concentration showed the greatest reduction at $\mathrm{V} 2\left(R^{2}=0.579 ; P<\right.$ 0.001), whereas there was no correlation between these variables in controls $\left(R^{2}=0.023 ; P=0.548\right)$. Twelve weeks consumption of $250 \mathrm{ml}$ per $\mathrm{d}$ of $\mathrm{OJ}$ did not adversely affect insulin sensitivity, circulating lipids or body weight.

Received 10th January 2016,

Accepted 7th March 2016

DOI: $10.1039 / \mathrm{c} 6 f \circ 00039 \mathrm{~h}$

www.rsc.org/foodfunction

\section{Introduction}

With the current global epidemic of obesity and associated comorbidities, there is interest in the contribution of dietary factors to the development and alleviation of obesity and metabolic syndrome, including cardiovascular risk factors such as hypercholesterolemia and hypertriacylglycerolemia. Dysregulation of lipid and lipoprotein metabolism can contribute to the development of atherosclerosis and cardiovascular disease, ${ }^{1}$ and systematic reviews have indicated that improvement of the serum lipid profile can reduce mortality related to atherosclerosis. $^{2}$

Regular consumption of soda drinks containing high-fructose corn syrup and sucrose has been suggested as a risk

\footnotetext{
${ }^{a}$ University of Nottingham, School of Life Sciences, Queen's Medical Centre, Nottingham, NG7 2UH, UK. E-mail: liz.simpson@nottingham.ac.uk ${ }^{b}$ Nottingham Universities Hospital NHS Trust, Queen's Medical Centre, Nottingham, $N G 72 U H, U K$
}

factor in the development of obesity in children ${ }^{3}$ and some researchers have expressed concern that a high dietary intake of $100 \%$ juice may also contribute to the development of obesity and the metabolic syndrome in adults, the latter perhaps due to increased fructose consumption. ${ }^{4}$ However, a relationship between $100 \%$ juice consumption and adverse metabolic consequences is not universally accepted, ${ }^{5,6}$ with epidemiological surveys, such as the NHANES, suggesting that consumption of fruit juice is associated with lower BMI and reduced indices of the metabolic syndrome, including circulating total cholesterol concentration. ${ }^{7,8}$ Despite the sugars content of $100 \%$ juice, it is proposed that characteristics of fruit juice, not found in sweetened beverages, in particular phenolic compounds and flavonoids, may be protecting individuals from adverse metabolic effects. Indeed, lower fasting total and Low Density Lipoprotein (LDL) cholesterol, and Apolipoprotein B (Apo-B) have been observed in daily consumers of orange juice compared with nonconsumers. ${ }^{9}$ 
In vitro studies, using isolated liver cells, have shown that citrus flavonoids can reduce net Apo-B secretion, by inhibiting synthesis of the cholesterol esters required for LDL production. ${ }^{10}$ This LDL lowering effect of purified citrus flavonoids is supported by in vivo supplementation studies in rodents, ${ }^{11-13}$ rabbits, ${ }^{14}$ and humans, ${ }^{15}$ and the presence of these flavonoids in orange juice (the most commonly consumed $100 \%$ juice in the US) may contribute to the observation of reduced serum total cholesterol concentration measured in epidemiological studies. ${ }^{8}$ However, prospective data from humans supplementing their diet with $100 \%$ orange juice are limited and equivocal. Short-term, high-dose consumption (750 $\mathrm{ml} \mathrm{d}^{-1}$ for 4-8 weeks), in hypercholesterolemic patients, has been shown to lower serum LDL concentrations ${ }^{16}$ or have no effect on this parameter, ${ }^{17}$ with high-density lipoprotein cholesterol (HDL) concentration in these patients being unaffected by supplementation in the former study ${ }^{16}$ but increased in the latter. ${ }^{17}$ However, the above studies were potentially compromised by the lack of blinding, supplementation in conjunction with other dietary advice, and the absence of a placebo control drink. Moreover, supplementing the diet with $750 \mathrm{ml}$ of orange juice per day, is equivalent to adding 5 UK recommended portions of $100 \%$ juice, ${ }^{18}$ and provides approximately $60 \mathrm{~g}$ of sugars plus an extra $1 \mathrm{MJ}$ in dietary energy intake. Increasing dietary intake of sugars, when in positive energy balance, has been associated with detrimental changes to the circulating lipid profile in humans. ${ }^{19}$ It is therefore important for any lipid-modifying effects of orange juice consumption to be assessed prospectively at a daily intake which is more representative of UK consumption guidelines. Kurowska et al. did not observe any statistical improvement in blood lipid profile after a month's supplementation with $250 \mathrm{ml}$ of orange juice a day. ${ }^{17}$ However, a $5 \%$ increase in circulating HDL concentration was observed, which may be further increased over a longer time frame.

The current study investigated the effect of 3 month's daily consumption of $250 \mathrm{ml}$ of orange juice on lipid profile, body weight and fasting insulin sensitivity in overweight men with elevated serum total cholesterol concentration. A sugars- and energy-matched control drink was used to standardise for any potential confounding effect of increasing dietary sugars and energy intake on variables, and to investigate any positive effects that may be associated with orange juice consumption.

\section{Results}

\section{Participants}

Initial interest was expressed by 280 men, 71 of whom fulfilled inclusion criteria (except elevated total serum cholesterol concentration), and undertook a medical screening. In total, 36 of these individuals were suitable to participate and were randomly assigned into either the orange juice (OJ) or control (CON) group, with groups being matched for age (OJ: 48.3(3.9) y, CON: $48.9(4.3) \mathrm{y} ; P=0.627)$. No participants withdrew from the study.

\section{Anthropometry}

Groups were matched at baseline with respect to body weight $(P=0.988)$, and the intervention did not result in a change in this variable (Table $1 ; P=0.807$ ). At the start of the study, all participants were either overweight or grade 1 obese (OJ: 29.9 (2.3) $\mathrm{kg} \mathrm{m}^{-2}$, CON: 29.3 (1.7) $\mathrm{kg} \mathrm{m}^{-2}$ ), with mean BMI being similar between the groups $(P=0.370)$. Waist and hip circumference and $\%$ total body fat of the participants reflect the overweight nature of the group, and the group mean waist : hip ratios were at values reported to confer an increased risk of developing cardiovascular disease. ${ }^{20}$ These variables were matched between groups at visit 1 (waist: $P=0.560$; hip: $P=0.541$; waist : hip ratio: $P=0.971 ; \%$ total body fat: $P=$ 0.792 ) and were unaffected by the intervention (waist: $P=$ 0.619 ; hip: $P=0.760$; waist : hip ratio: $P=0.888$; $\%$ total body fat: $P=0.556)$.

\section{Fasting insulin sensitivity}

Participants were not recruited on the basis of demonstrating insulin resistance, (with respect to fasting glucose and insulin concentration). However, as with other overweight cohorts reported in the literature, HOMA-IR values indicative of fasting insulin resistance were observed (Table 1). Groups were matched for HOMA-IR pre-intervention $(P=0.546)$ and this measure did not change as a result of the supplementation period (OJ: $P=0.586, \mathrm{CON}: P=0.609)$.

Table 1 Mean (unless otherwise indicated) values for anthropometric, BP and fasting insulin resistance parameters. Mean data display the SD in parentheses and median data show the 25th and 75th percentile in parentheses. Comparison of groups at baseline, and the change seen after the intervention, between groups, was $P>0.05$ for each variable

\begin{tabular}{|c|c|c|c|c|}
\hline & \multicolumn{2}{|l|}{ OJ $(n=18)$} & \multicolumn{2}{|l|}{$\operatorname{CON}(n=18)$} \\
\hline Waist circumference $(\mathrm{cm})$ & $105.1(6.16)$ & $0.06(2.80)$ & $104.1(4.47)$ & $-0.44(3.17)$ \\
\hline Hip circumference $(\mathrm{cm})$ & $109.8(5.36)$ & $0.39(5.03)$ & $108.8(4.59)$ & $-0.03(2.76)$ \\
\hline Waist : hip & $0.96(0.05)$ & $-0.003(0.04)$ & $0.96(0.04)$ & $-0.005(0.03)$ \\
\hline Total body fat (kg) & $26.39(5.86)$ & $-0.19(2.24)$ & $28.82(4.94)$ & $-0.39(1.78)$ \\
\hline
\end{tabular}




\section{Incretin hormones and leptin}

Circulating PYY, Ghrelin, GLP-1 and leptin concentration, when fasted, were similar between the groups at V1 $(P=0.673$, $0.767,0.481$ and 0.839 respectively; Table 2$)$. No changes to PYY $(P=0.500)$, ghrelin $(P=0.472)$ or leptin $(P=0.112)$ occurred as a result of OJ, although there was a trend for GLP-1 to be reduced in this group $(P=0.081)$. In CON, no changes in these variables were observed (PYY: $P=0.616$, ghrelin: $P=0.420$, GLP-1: $P=0.760$, leptin: $P=0.360)$. When OJ was compared to CON, no intervention effect of orange juice consumption was observed for PYY $(P=0.815)$, ghrelin $(P=0.279)$, or GLP-1 response $(P=0.226)$, although there was a weak trend for the change in leptin to be different $(P=0.097)$.

\section{Inflammatory markers and uric acid}

At V1, circulating TNF $\alpha$ was significantly higher $(P=0.034)$, CRP markedly lower $(P=0.019)$ and a trend for IL-6 to be higher $(P=0.083)$ in the OJ group (Table 2). However, these variables did not change as a result of either intervention ( $P \geq 0.312$ in each case) and there was no difference in the changes at week 12 between groups (TNFo: $P=0.959$, IL-6: $P=$ 0.987 and CRP: $P=0.118)$. Fasting uric acid concentration was similar between groups at V1 $(P=0.661)$ and did not change as a result of the interventions (OJ: $P=0.179$, CON: $P=0.813$ ). Moreover, no difference in UA response to the interventions, between groups was noted $(P=0.303)$.

\section{Lipids}

Participants were selected on the basis of having a total cholesterol concentration of between 5 and $7 \mathrm{mmol} \mathrm{l}^{-1}$, and this was reflected in the mean fasting total cholesterol values being above the healthy range, but below a concentration which would require therapeutic intervention. The 2 groups were similar with regards to serum lipid and lipoprotein concentrations pre-intervention, including; total cholesterol $(P=$ $0.207)$, LDL $(P=0.085)$, HDL $(P=0.839)$, NEFA $(P=0.805)$, ApoA1 $(P=0.963)$ and Apo-B $(P=0.126$; Table 3$)$. These parameters did not change significantly across visits within groups, and no differences were observed between groups, over the intervention period $(P>0.05)$.

Although median TAG concentration of both groups was comparable at V1 $(P=0.839)$ and V2 $(P=0.613)$, there was a trend for the change in this variable to be different between the groups after the intervention period $(P=0.060)$, with fasting TAG being significantly higher at V2 in controls $(P<0.05)$. Although median TAG was numerically lower in the OJ group at V2, compared with V1, this was not statistically significant or notable in terms of a trend. However, in the OJ group, those with the highest initial triacylglycerol concentration pre-intervention, showed the greatest reduction after 12 weeks supplementation, whereas there was no correlation between these variables in the control group (Fig. 1). There were also no significant associations observed between the change in dietary carbohydrate, total sugars or energy intake

Table 2 Median (unless otherwise indicated) concentration of circulating variables sampled in the fasted state before and at week 12 of the intervention. Median data show the 25th and 75th percentile in parentheses and mean data display the SD in parentheses

\begin{tabular}{lccccc}
\hline & OJ $(n=18)$ & & CON $(n=18)$ \\
\cline { 2 - 3 } & Pre-intervention & Change at week 12 & & Pre-intervention & Change at week 12 \\
\hline PYY $\left(\mathrm{pg} \mathrm{ml}^{-1}\right)$ & $86.8(77.8-107.6)$ & $6.7(-9.5-13.7)$ & & $99.1(71.0-118.8)$ & $0.7(-7.9-12.6)$ \\
Ghrelin $\left(\mathrm{pg} \mathrm{ml}^{-1}\right)$ & $797.9(722.7-1096.8)$ & $-9.1(-62.5-117.3)$ & & $933.7(763.7-1056.9)$ & $25.0(-103.9-62.4)$ \\
GLP-1 $\left(\mathrm{pmol} \mathrm{l}^{-1}\right)$ & $2.24(1.94-4.00)$ & $-0.14(-0.77-0.43)$ & & $2.32(1.16-3.49)$ & $-0.02(-0.43-0.56)$ \\
Leptin $\left(\mathrm{ng} \mathrm{ml}^{-1}\right)$ & $7.88(6.81-14.36)$ & $-0.58(-1.99-0.57)$ & & $8.23(6.88-12.62)$ & $0.32(-1.03-1.85)$ \\
CRP $\left(\mathrm{pg} \mathrm{ml}^{-1}\right)$ & $0.91^{a}(0.48-1.77)$ & $0.13(-0.21-0.66)$ & & $1.86(1.08-3.74)$ & $0.01(-1.29-0.90)$ \\
IL-6 $\left(\mathrm{pg} \mathrm{ml}^{-1}\right)$ & $3.93(2.23-5.94)$ & $0.00(-0.94-0.82)$ & & $2.33(2.09-3.27)$ & $0.00(-0.95-1.01)$ \\
TNFo $\left(\mathrm{pg} \mathrm{ml}^{-1}\right)$ & $3.24^{a}(2.34-3.88)$ & $-0.06(-0.84-0.41)$ & & $2.22(1.69-3.01)$ & $-3.00(-0.99-0.89)$ \\
Mean UA $\left(\mu \mathrm{mol} \mathrm{l}^{-1}\right)$ & $376.7(72.54)$ & $15.6(47.10)$ & $387.1(68.07)$ &
\end{tabular}

${ }^{a} P<0.05$ compared to control group at pre-intervention.

Table 3 Lipid parameters. Data are the median with the 25th and 75th percentile displayed in parentheses. Comparison of groups at baseline, and the change seen after the intervention, between groups, was $P>0.05$ for each variable

\begin{tabular}{|c|c|c|c|c|}
\hline & \multicolumn{2}{|l|}{$\mathrm{OJ}(n=18)$} & \multicolumn{2}{|l|}{$\mathrm{CON}(n=18)$} \\
\hline & Pre-intervention & Change at week 12 & Pre-intervention & Change at week 12 \\
\hline Total cholesterol $\left(\mathrm{mmol} \mathrm{l}^{-1}\right)$ & $5.62(5.22-6.53)$ & $0.02(-0.54-0.36)$ & $6.21(5.78-6.63)$ & $0.23(-0.32-0.33)$ \\
\hline $\operatorname{LDL}\left(\mathrm{mmol} \mathrm{l}^{-1}\right)$ & $3.64(3.38-4.24)$ & $0.04(-0.33-0.33)$ & $4.20(3.65-4.53)$ & $-0.01(-0.41-0.22)$ \\
\hline $\mathrm{HDL}\left(\mathrm{mmol} \mathrm{l}^{-1}\right)$ & $1.11(1.03-1.34)$ & $-0.02(-0.11-0.08)$ & $1.17(1.04-1.37)$ & $-0.02(-0.05-0.03)$ \\
\hline $\operatorname{NEFA}\left(\mathrm{mmol} \mathrm{l}^{-1}\right)$ & $0.39(0.32-0.56)$ & $-0.08(-0.19-0.02)$ & $0.44(0.31-0.55)$ & $-0.02(-0.18-0.08)$ \\
\hline Apo-A1 $\left(\mathrm{g} \mathrm{l}^{-1}\right)$ & $1.23(1.10-1.28)$ & $0.06(-0.04-0.09)$ & $1.22(1.10-1.32)$ & $0.01(-0.04-0.08)$ \\
\hline Аро-В $\left(\mathrm{g} \mathrm{l}^{-1}\right)$ & $1.08(1.04-1.26)$ & $0.06(-0.10-0.10)$ & $1.27(1.10-1.36)$ & $0.02(-0.11-0.07)$ \\
\hline TAG $\left(\mathrm{mmol} \mathrm{l}^{-1}\right)$ & $1.52(0.93-2.30)$ & $-0.11(-0.68-0.53)$ & $1.47(1.04-2.60)$ & $0.29(-0.07-0.72)$ \\
\hline
\end{tabular}




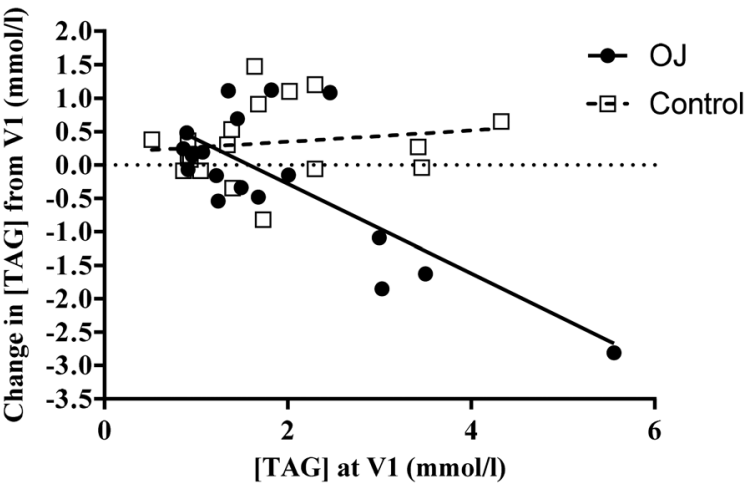

Fig. 1 Correlation between pre-intervention TAG concentration (at V1) and change in TAG concentration (from V1) over the 12 week intervention ( $n=18$ in each group). OJ group $R^{2}=0.579(P<0.001)$, control group $R^{2}=0.023(P=0.548)$.

after 12 weeks supplementation, and the change in serum TAG concentration, in either group. Spearman's rho was $0.345(P=$ $0.227), 0.213(P=0.464)$, and $0.165(P=0.573)$ respectively in the OJ participants, and $0.016(P=0.957),-0.066(P=0.831)$ and $-0.462(P=0.112)$ respectively in the control group. Indeed, the trend for the change in TAG to be different, between the groups, after the intervention period, remained when sugars intake at V1 was used as a covariate $(P=0.076)$. However, when either dietary energy or carbohydrate intake at V1 were used as a covariate, this trend was no longer present $(P=0.110$ and $P=0.103$, respectively).

\section{Dietary intake}

Complete diet diaries were obtained for 33 out of 36 participants (Table 4). When compared with CON, those in the OJ group reported a greater intake of carbohydrate $(P<0.005)$ and total sugars $(P<0.01)$ before the intervention period, and this was reflected in the latter showing higher daily energy intake $(P<0.05)$. Indeed, at baseline, total sugars contributed a greater percentage of daily energy in the OJ group, compared with the control group $(P<0.05)$. However, despite daily consumption of OJ, the amount of sugars consumed and percentage of dietary energy intake derived from total sugars reported at week 11 did not change $(P=0.686$ and $P=0.950$ respectively), suggesting that participants in this group were compensating for the extra $22 \mathrm{~g}$ of total sugars provided by the orange juice, by reducing consumption of other products containing sugars. Indeed, daily energy intake at week 11 in this group was reduced compared to pre-intervention $(P<0.05)$, although the macronutrient composition of the diet did not appear to be affected by this reduction in energy intake. In the control group, neither reported daily energy intake, nor macronutrient composition of the diet changed as a result of the intervention $(P>0.05$ in each case; Table 4$)$. However, there was a difference, between groups, in the effect of the interventions on both daily intake of carbohydrate and proportion of total energy provided by carbohydrate $(P<0.05)$.

\section{Discussion}

Media reports have claimed that regular 100\% juice consumption adversely effects body weight, insulin sensitivity and blood lipid profile. This study did not observe detrimental consequences of 12 weeks daily orange juice consumption in any of these parameters. Review of fructose, and other simple sugars, feeding studies suggests that it is likely to be the overfeeding of energy which can occur with supplementation protocols, rather than ingestion of the sugars per se which confers detrimental metabolic effects. ${ }^{19,21}$ In the current study, participants appeared to be in energy balance, as body weight did not increase as a result of either intervention. The absence of an increase in body weight has been noted in other studies supplementing the diet with citrus juice, ${ }^{16,17,22}$ although $100 \%$ juice consumption does not appear to suppress appetite to a greater extent than non-juice beverages containing sugars, ${ }^{23}$ and in the current study convincing evidence for increased satiety signalling, based on fasting incretin hormone profiles in the 2 groups, was not observed.

Table 4 Mean (unless otherwise indicated) reported daily macronutrient intake and macronutrient composition of the diet (expressed as \% of total energy intake) for 33 participants. Median data show the 25th and 75th percentile displayed in parentheses, and mean data display the SD in parentheses

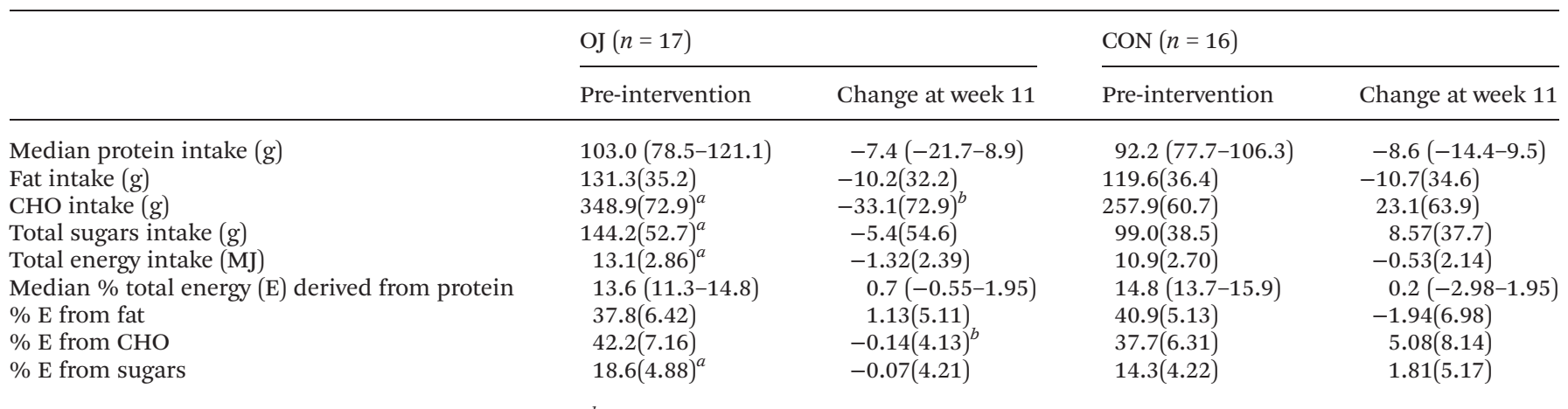

${ }^{a} P<0.05$ compared to control group pre-intervention. ${ }^{b} P<0.05$ compared to control group, for change at week 11. 
Strengths of the current study were the use of an energy and sugars matched control drink (to isolate any difference between $100 \%$ juice and a sweetened beverage) and a singleblinded study design (to identify any placebo effect of participating in the study). Participants were not aware that $100 \%$ juice was being investigated and did not know whether they had been randomised to the drink containing citrus flavonoids or no citrus flavonoids. In previous studies examining the effect of OJ consumption on lipid parameters, participants were not blinded to the drink being consumed, which may have altered their health behaviours and had an impact on outcomes. However, in the current study, 12 weeks daily supplementation of the diet with the control drink did not result in significant increases in body weight, insulin resistance, or circulating cholesterol. It is therefore not possible to determine whether phenolic compounds and flavonoids found in fruit juice can protect individuals from these purported adverse metabolic effects of consuming drinks containing sugars.

In the present study, components within orange juice may have mitigated the negative effect of regular sweet drink consumption on circulating TAG concentration, and may have promoted a reduction in this variable in those who demonstrated hypertriacylglycerolemia at V1. Although the observed effects of interventions on circulating TAG are statistically underpowered, the impact that $\mathrm{OJ}$ consumption could be having on TAG concentration in the current study supports findings from rodent and human studies which have supplemented the diet with purified flavonoids. ${ }^{13,24}$ However, observations are not consistent with other human in vivo studies supplementing with whole juice, which have observed no change in TAG concentration after 4 weeks when up to $500 \mathrm{ml}$ per d of orange juice have been provided, ${ }^{17,22}$ but an increase when participants consumed $750 \mathrm{ml} \mathrm{d}{ }^{-1} \cdot{ }^{17}$ Our data and that of Miwa et l $^{24}$ suggest that the lowering effect of orange juice, or citrus flavonoid, supplementation on circulating TAG is not observed where plasma TAG concentration is within the healthy range, and might explain why improvements in TAG concentration were not seen in normotriacylglycerolemic individuals in the Morand study. ${ }^{22}$

A criticism of the current study is that despite a randomised design, the OJ group reported greater carbohydrate, total sugars and dietary energy intake than the control group before the intervention began, and covariate analysis suggested that carbohydrate and/or energy intake at V1 may have had an impact on the subsequent response of circulating TAG to the interventions. Increasing the proportion of carbohydrate in the diet has been associated with a rise in plasma TAG concentration, ${ }^{25}$ and in the current study the change in dietary carbohydrate intake at week 11 of supplementation in the OJ group, compared with that in controls, may have contributed to the observed trend for the change in circulating TAG to be different at V2 between groups. Furthermore, although there appears to be a genetic component to this observation, overfeeding energy has also been shown to increase plasma TAG concentration $^{26}$ and the reduction in dietary energy intake observed in the OJ group may have had an impact on this variable. However, overfeeding is generally accompanied by modifications to other lipid measures, such as increased LDL and ApoB, and decreased $\mathrm{HDL},{ }^{26}$ and changes to these variables were not observed. Indeed, changes in reported energy intake were not reflected in any significant alterations to body weight in these cohorts, and it is possible that recording dietary intake over 3 days was not sufficient to truly reflect the habitual diet, as has been reported by others. ${ }^{27}$ Further investigation, with more detailed dietary intake measures, is required to determine the potential confounding effect of macronutrient composition and energy balance on the TAG modifying effects of orange juice supplementation.

Previous studies have reported a LDL-lowering effect of orange juice and citrus flavonoid supplementation in man, ${ }^{15,24,28}$ but this is not a universal finding. ${ }^{17,29}$ Indeed, no change in LDL concentration was observed in the current study. Data from in vitro research suggest that the LDL modifying ability of citrus juices is mediated through their flavonoid components. ${ }^{10}$ It is therefore possible that the failure to improve LDL profile in the current investigation was due to the amount of flavonoids provided being too low; studies reporting improvements in LDL concentration with daily intake of purified flavonoids, have supplemented the diet of participants with approximately $0.7 \mathrm{mmol}$ of glucosyl hesperidin or naringin (naringenin 7-O-rhamnoglucoside), ${ }^{15,24}$ whereas the amount provided by the orange juice in the present study was approximately $0.22 \mathrm{mmol}$ of hesperidin and $0.03 \mathrm{mmol}$ of narirutin (naringenin-7-O-rutinoside) per day. However, data in the literature do not provide evidence for this supposition. The reported quantity of flavonoids provided by daily consumption of $750 \mathrm{ml}$ of orange juice, in a study which induced a reduction in LDL concentration, was of a similar magnitude to that provided in the current study $(0.14 \mathrm{mmol}$ hesperitin and $0.02 \mathrm{mmol}$ naringin) ${ }^{16}$ and daily supplementing the diet with greater amounts $(1.3 \mathrm{mmol}$ hesperidin or $0.86 \mathrm{mmol}$ naringin) did not result in any improvements to circulating lipids in those with elevated cholesterol. ${ }^{29}$ It is therefore difficult to identify a clear explanation for the differences in LDL response to citrus juice and purified citrus flavonoid supplementation seen in the in vivo human studies, but statistical power, variability in study design (including absence of placebo control), quantity of juice or flavonoids used, supplementation durations, character of participant cohorts, habitual diet, fibre content, or the forms of citrus flavonoids being used (glycoside $v s$. aglycone), may all play a part. Further investigation of these confounding factors may help to clarify any potential health benefit of citrus consumption.

\section{Experimental}

\section{Trial design}

A randomised, single-blinded, placebo controlled, parallel group study design was used. The random allocation sequence was generated using an on-line calculator ${ }^{30}$ with the number 
of individuals in each group balanced in 3 blocks (12 individuals per block; 6 OJ and 6 control). Blocks were used to promote participants of each group being on the trial at similar times of the year, to mitigate any confounding effects of seasonal variation in habitual diet. Individuals were randomised at the point of entering the study, with their participant number allocated sequentially.

This study was conducted according to the guidelines laid down in the Declaration of Helsinki and all procedures involving human subjects were approved by the University of Nottingham Medical School Ethics Committee. Written informed consent was obtained from all subjects, and the protocol was registered at http://www.clinicaltrials.gov reference NCT01350843.

\section{Participants}

Thirty-six overweight, or mildly obese men (BMI 27-35 $\mathrm{kg} \mathrm{m}^{-2}$; aged 40-60 years) who were otherwise healthy, were recruited from the general population of Nottinghamshire, UK. All participants attended the David Greenfield Human Physiology Unit (Queen's Medical Centre, Nottingham, UK) for an initial medical screening visit to confirm health status. In addition, blood pressure (BP) was measured at the left upper arm (after lying semi-supine for $5 \mathrm{~min}$ ) using automated oscillometry (Dynamap Pro 1000; GE Medical, Milwaukee, USA). BMI was calculated from measured height and weight. If participants were suitable to take part with regards to their health questionnaire and BMI measures, a venous blood sample was then taken to assess fasting lipid concentration. Those taking lipidlowering medication, or who reported using nutritional strategies to lower their cholesterol, were excluded. Participants were accepted onto the trial if their serum total cholesterol concentration was between $5-7 \mathrm{mmol} \mathrm{l}^{-1}$, they did not consume citrus juice or whole fruit on a daily basis, they did not have any food allergies related to the investigational product, and did not demonstrate any clinically significant abnormalities on screening. Eligibility criteria were not altered during the recruitment period.

\section{Intervention}

After recruitment, participants were asked to record all food intake (including snacks and drinks) in a diet diary for 3 days ( $2 \times$ week or work days, and $1 \times$ weekend or rest day) for baseline assessment of macronutrient and energy intake, and to attend the David Greenfield Human Physiology Unit for 2 further visits (at weeks 0, and 12). These visits took place in the morning after the individual had fasted from midnight the night before. Initially, measurement of body mass was made to the nearest $0.1 \mathrm{~kg}$ using a Seca 882 Digital Scale (Birmingham, UK), and waist and hip circumference were assessed (with the participant standing) mid-way between the lowest rib and the iliac crest, and at the greater trochanters, respectively. Participants were then asked to rest, semi-supine, on a couch for $5 \mathrm{~min}$ before having their resting $\mathrm{BP}$ measured, as previously described. A fasting blood sample was taken for determination of serum insulin, leptin, uric acid (UA), TNF $\alpha$,
C-Reactive Protein (CRP), interleukin-6 (IL-6), total cholesterol, HDL, LDL, apolipoprotein A1(Apo-A1), and Apo-B, plasma PYY, ghrelin, glucagon-like protein (GLP-1), TAG and non-esterified fatty acids (NEFA), and whole blood glucose. Whole blood glucose was immediately assessed using the glucose hydrogenase photometric method (Hemocue, Agelholm, Sweden; within assay precision being $1.41 \%$ ), with other samples frozen at $-80{ }^{\circ} \mathrm{C}$ and stored until analysis at a later date.

Participants were asked to consume $250 \mathrm{ml}$ of either orange juice (as frozen concentrate orange juice; FCOJ, provided by Florida State Department of Citrus, USA, and diluted 1 part concentrate to 3 parts water before consumption; FCOJ was $42^{\circ}$ Brix and reconstituted OJ was $11.8^{\circ}$ Brix), or an energy and sugars matched, orange-flavoured control drink (MPBioscience Ltd, Derby, UK), once a day for 12 weeks. Participants in the OJ group were provided the FCOJ, a measuring jug and storage vessel for the reconstituted drink. Instructions on how to reconstitute the juice before consumption and how to store both the frozen and reconstituted drink were also provided. The quantity consumed was chosen to reflect the average volume of $100 \%$ juice portions available commercially in the UK (which range from $200 \mathrm{ml}-330 \mathrm{ml}$ ), and allow comparison with previous studies. A sugars matched control was selected to identify any confounding effects that supplementing the diet with additional sugars and energy may have. The composition of the drinks is shown in Table 5, and the citrus flavonoids contained in the orange juice reflect their natural abundance in the product.

The 12 week supplementation period began on the day after the first study visit. In all documentation and interaction with participants, the products were described as an 'orange flavoured drink' which was either rich in citrus polyphenols or low in these compounds. The study was therefore single blinded; those providing drink supplies to participants knew which product participants received, but participants and those executing the biochemical analysis were blinded. Weekly telephone contact with the study participants was maintained over the dosing period to improve compliance. No problems with the supplementation protocol or the palatability of the drinks were reported by participants.

Individuals were asked to complete a further 3-day diet diary (as previously described) in the week before the second study visit (week 11), to assess any changes in macronutrient or energy intake which may have occurred as a consequence of the intervention. Household measures were used to estimate

Table 5 Composition of drinks, with regards to sugars, vitamin $\mathrm{C}$ and citrus flavonoids contained in one $250 \mathrm{ml}$ serving

\begin{tabular}{lcc}
\hline & Orange juice & Control drink \\
\hline Sucrose $(\mathrm{g})$ & 10.78 & 11.66 \\
Fructose $(\mathrm{g})$ & 5.60 & 5.87 \\
Glucose $(\mathrm{g})$ & 5.25 & 4.95 \\
Vitamin C (mg) & 137 & 90 \\
Hesperidin (mg) & 135.4 & 0 \\
Narirutin (mg) & 15.5 & 0
\end{tabular}


portion size, and diaries were subsequently analysed using a food composition database (WISP V2, Tinuviel Software UK 2003). To calculate habitual diet composition, a mean daily intake was obtained from all 3 days of each recording period, and macronutrient composition was expressed as a percentage of total energy intake. These data were combined to produce group means.

\section{Analytical methods}

Serum insulin was assessed using a human-specific radioimmunoassay (Merck Millipore, Billerica, MA, USA), with an intra-assay coefficient of variation (CV) of $4.41 \%$, and an interassay CV of $10.53 \%$. NEFA was analysed by the ACS-ACOD method (Wako Diagnostics, Richmond, VA, USA), and had an intra-assay CV of $0.65 \%$, an inter-assay CV of $2.95 \%$. TAG, total cholesterol, LDL and HDL were measured using enzymic photometric methods (Horiba Medical, Montpellier, France), and the intra-assay CVs were $2.16 \%, 2.63 \%, 2.21 \%$ and $0.77 \%$ respectively, with inter-assay CVs being 3.27\%, 2.6\%, 4.77\% and $4.72 \%$. Apo-A1 and Apo-B were analysed using enzymic turbidimetric methods (Horiba Medical, Montpellier, France) and within assay precision for these assays were $0.86 \%$ and $1.96 \%$ with between assay precision being $1.33 \%$ and $1.96 \%$, respectively.

\section{Statistical methods}

All data were coded and analysed using SPSS version 16.0 (Statistical Package for the Social Sciences 2000). Data were initially checked for normality of distribution (using criteria of skewness or kurtosis $z$-score between -1.96 and 1.96). Parametric data are described in the text and tables as the mean with the standard deviation in parentheses, with non-parametric data displayed as the median with the $25 \%$ to $75 \%$ quartile range shown in parentheses. Group characteristics at baseline were compared between groups using unpaired students $t$-test or non-parametric equivalent (Mann-Whitney $U$ ) as appropriate. Within group comparisons of variables measured at baseline (week 0) and at the end of intervention (week 12), were investigated using paired Student's $t$-test, or a non-parametric equivalent (Wilcoxon signed ranks). To assess the effect of intervention on variables, a mixed model ANOVA with repeated measures was used. Violation of the assumption of sphericity was assessed using Mauchly's test, and where appropriate the Greenhouse-Geisser statistic was used to determine the $P$ value. All analysis used a two-tailed assessment and statistical significance was assumed where $P<0.05$.

\section{Sample size}

Using data generated from the Cesar et al. (2010) to inform the variability of LDL-cholesterol concentration in a hypercholesterolemic population, and a parallel study design with 18 in each group (OJ vs. carbohydrate matched control), this would give the statistical power to detect a difference in mean LDL cholesterol of $0.5 \mathrm{mmol} \mathrm{l}^{-1}\left(21 \mathrm{mg} \mathrm{dl}^{-1}\right)$, which is the magnitude of change reported in hypercholesterolemic patients after orange juice consumption. ${ }^{16}$

\section{Conclusions}

Daily consumption of $250 \mathrm{ml}$ of orange juice for 3 months did not result in an increase in dietary sugars intake in a cohort of overweight men with elevated total cholesterol concentration, and despite media concern, an increase in body weight or decreased insulin sensitivity did not occur over the intervention. Moreover, regular orange juice consumption did not adversely affect fasting blood lipids, and may help reduce elevated plasma triacylglycerol concentration. However, changes in carbohydrate and/or dietary energy intake observed during the supplementation period are likely to have confounded this latter observation.

\section{Conflicts of interests}

Florida Department of Citrus funded this study and provided the orange juice used for supplementing the diet. Study participants and the Ethics Committee were made aware of the source of funding for this study. The study sponsor approved the study design, but had no involvement with data collection, analysis, or interpretation. Moreover, the sponsor did not influence the writing of the report or the decision to submit the report for publication.

IAM is on Scientific Advisory Boards for Nestlé, Ikea and Mars Inc.

\section{Acknowledgements}

Experimenters would like to thank Mr I. H. Bennett for subject recruitment and assisting with data collection, Mrs S. Cordon and Mrs K. Swift for carrying out the biochemical analysis, Miss M. Marshall for analysing the diet diaries, and Florida State Department of Citrus for providing the orange juice.

The work was supported by an infrastructure award from MRC/ARUK to establish a centre of excellence in musculoskeletal ageing research (Grant No. MR/K00414X/1 and 19891 2012-2017).

\section{References}

1 J. M. Assini, E. E. Mulvihill and M. W. Huff, Curr. Opin. Lipidol., 2013, 24, 34-40.

2 F. Taylor, M. D. Huffman, A. F. Macedo, T. H. Moore, M. Burke, G. Davey Smith, K. Ward and S. Ebrahim, Cochrane Database Syst. Rev., 2013, 1, CD004816.

3 B. A. Swinburn, I. Caterson, J. C. Seidell and W. P. James, Public Health Nutr., 2004, 7, 123-146.

4 L. A. Bazzano, T. Y. Li, K. J. Joshipura and F. B. Hu, Diabetes Care, 2008, 31, 1311-1317.

5 K. Fujioka, F. Greenway, J. Sheard and Y. Ying, J. Med. Food, 2006, 9, 49-54.

6 C. O'Neil and T. Nicklas, Am. J. Lifestyle Med., 2008, 2, 315354. 
7 I. I. I. V. L. Fulgoni and M. A. Pereira, FASEB J., 2009, 23, LB506.

8 C. E. O'Neil, T. A. Nicklas, G. C. Rampersaud and V. L. Fulgoni, 3rd, Nutr. J., 2012, 11, 107.

9 N. P. Aptekmann and T. B. Cesar, Lipids Health Dis., 2013, $12,119$.

10 N. M. Borradaile, K. K. Carroll and E. M. Kurowska, Lipids, 1999, 34, 591-598.

11 R. W. Li, A. G. Theriault, K. Au, T. D. Douglas, A. Casaschi, E. M. Kurowska and R. Mukherjee, Life Sci., 2006, 79, 365373.

12 S. H. Bok, S. H. Lee, Y. B. Park, K. H. Bae, K. H. Son, T. S. Jeong and M. S. Choi, J. Nutr., 1999, 129, 1182-1185.

13 E. M. Kurowska and J. A. Manthey, J. Agric. Food Chem., 2004, 52, 2879-2886.

14 L. Hooper, P. A. Kroon, E. B. Rimm, J. S. Cohn, I. Harvey, K. A. Le Cornu, J. J. Ryder, W. L. Hall and A. Cassidy, Am. J. Clin. Nutr., 2008, 88, 38-50.

15 U. J. Jung, H. J. Kim, J. S. Lee, M. K. Lee, H. O. Kim, E. J. Park, H. K. Kim, T. S. Jeong and M. S. Choi, Clin. Nutr., 2003, 22, 561-568.

16 T. B. Cesar, N. P. Aptekmann, M. P. Araujo, C. C. Vinagre and R. C. Maranhao, Nutr. Res., 2010, 30, 689-694.

17 E. M. Kurowska, J. D. Spence, J. Jordan, S. Wetmore, D. J. Freeman, L. A. Piché and P. Serratore, Am. J. Clin. Nutr., 2000, 72, 1095-1100.

18 NHS Choices, 5 A DAY portion sizes, http://www.nhs.uk/ Livewell/5ADAY/Pages/Portionsizes.aspx, Accessed 08/09/ 2013, 2013.

19 R. D. Johnston, M. C. Stephenson, H. Crossland, S. M. Cordon, E. Palcidi, E. F. Cox, M. A. Taylor, G. P. Aithal and I. A. Macdonald, Gastroenterology, 2013, 145, 10161025 e1012.

20 J. L. Megnien, N. Denarie, M. Cocaul, A. Simon and J. Levenson, Int. J. Obes. Relat. Metab. Disord., 1999, 23, 90-97.

21 J. L. Sievenpiper, R. J. de Souza, A. Mirrahimi, M. E. Yu, A. J. Carleton, J. Beyene, L. Chiavaroli, M. di Buono, A. L. Jenkins, L. A. Leiter, T. M. S. Wolever, C. W. C. Kendall and D. J. A. Jenkins, Ann. Intern. Med., 2012, 156, 291-304.

22 C. Morand, C. Dubray, D. Milenkovic, D. Lioger, J. F. Martin, A. Scalbert and A. Mazur, Am. J. Clin. Nutr., 2011, 93, 73-80.

23 G. H. Anderson, Int. J. Obes., 0000, 30, S52-S59.

24 Y. Miwa, H. Mitsuzumi, T. Sunayama, M. Yamada, K. Okada, M. Kubota, H. Chaen, Y. Mishima and M. Kibata, J. Nutr. Sci. Vitaminol., 2005, 51, 460470.

25 E. J. Parks and M. K. Hellerstein, Am. J. Clin. Nutr., 2000, 71, 412-433.

26 M. Terán-García, J.-P. Després, C. Couillard, A. Tremblay and C. Bouchard, Atherosclerosis, 2004, 173, 277-283.

27 P. P. Basiotis, S. O. Welsh, F. J. Cronin, J. L. Kelsay and W. Mertz, J. Nutr., 1987, 117, 1638-1641.

28 T. B. Cesar, N. P. Aptekmann, M. P. Araujo, C. C. Vinagre and R. C. Maranhão, Nutr. Res., 2010, 30, 689-694.

29 I. Demonty, Y. Lin, Y. E. Zebregs, M. A. Vermeer, H. C. van der Knaap, M. Jakel and E. A. Trautwein, J. Nutr., 2010, 140, 1615-1620.

30 G. E. Dallal, http:/www.randomization.com, Tufts University, 2013. 